Dominika Szoke*, Simone Caruso, Sara Pasqualetti, Elena Aloisio, Felicia Stefania Falvella, Alberto Dolci and Mauro Panteghini

\title{
Suppressing all test results in grossly hemolyzed samples: is this approach appropriate in every case?
}

https://doi.org/10.1515/cclm-2018-1071

Received October 1, 2018; accepted October 2, 2018; previously published online October 23, 2018

Keywords: gross hemolysis; in vivo hemolysis; sample rejection.

To the Editor,

We read with great interest the article by Lippi et al. [1] reporting practical recommendations for managing hemolyzed samples on behalf of the European Federation of Clinical Chemistry and Laboratory Medicine (EFLM) Working Group for Preanalytical Phase (WG-PRE). We were particularly interested in the application of one of the recommendations, namely, the number 5 , i.e. if cell-free hemoglobin concentration (free $\mathrm{Hb}$ ) in the collected blood sample is $>10 \mathrm{~g} / \mathrm{L}$, all laboratory results should be suppressed and replaced with a comment, indicating that data cannot be released because the sample is grossly hemolyzed.

Because hemolytic samples are in $>95 \%$ of cases due to in vitro hemolysis, laboratory results obtained on such specimens and reported to clinical wards would be "erroneous" if hemolysis interferes in analytical measurements [2]. On the other hand, whenever in vivo hemolysis is reasonably suspected, test results, except those analytically interfered (for information on the mechanisms of interference, see Lippi et al. [2]), should be released by the laboratory, as the information can be helpful for the patient treatment and the identification of the underlying cause, which is frequently a life-threatening condition.

*Corresponding author: Dominika Szoke, UOC Patologia Clinica, ASST Fatebenefratelli-Sacco, Via GB Grassi 74, 20157 Milano, Italia, Phone: +39 023904 2683, Fax: +39 023904 2364,

E-mail: dominika.szoke@asst-fbf-sacco.it

Simone Caruso, Sara Pasqualetti, Elena Aloisio, Felicia Stefania

Falvella, Alberto Dolci and Mauro Panteghini: UOC Patologia Clinica, ASST Fatebenefratelli-Sacco, Milano, Italia
Sometimes the cause is obvious from the clinical history, but often the diagnosis of intravascular hemolysis requires a synthesis of all clinical information and appropriate laboratory tests [3]. Typical laboratory findings are an increase in reticulocyte count, abnormal bilirubin (unconjugated) and lactate dehydrogenase concentrations in blood and reduced serum haptoglobin, with presence in blood film of spherocytes, agglutination or polychromasia. In case of intravascular hemolysis leading to hemoglobinuria, urinalysis can be positive for hemoglobin, but urine microscopy can be negative for red cells, and urinary hemosiderin can be detected approximately 1 week after the onset.

Result processing in case of gross hemolysis is not a new topic [4], but the harmonization of the management of unreliable samples remains one of the most challenging issues in the integrated project of harmonization of hemolysis assessment [5]. In the era of total laboratory automation, which makes use of the automated measurement of serum interference indices, clinical laboratories can easily handle grossly hemolyzed samples in conformity with recommendations [1]. However, one of the unsolved problems is the identification and handling of samples where gross hemolysis can be due to in vivo causes. The percentage of in vivo hemolysis among hemolyzed samples of all degrees was found to be 3.2\% [4]. However, we were unable to find articles where the rate of in vivo hemolysis was estimated only in grossly hemolyzed samples (i.e. with free $\mathrm{Hb}>10 \mathrm{~g} / \mathrm{L}$ ). Our aim was, therefore, to evaluate how much frequent is in vivo hemolysis among grossly hemolyzed samples for which, according to the EFLM WG-PRE recommendations, all laboratory results should be suppressed.

In our clinical laboratory serving a 600-bed metropolitan university hospital, a 400-bed healthcare residence, and with a daily affluence of 650 outpatients, the quality of blood samples is systematically assessed by the automated measurement of interference indices in all incoming serum and plasma samples [6]. Over a 
1-year period (June 2017-May 2018), we retrieved clinical and laboratory data of all patients who had a blood sample (plasma [BD Vacutainer lithium heparin tubes, cat. no. 368884] or serum [BD Vacutainer silica gel tubes, cat. no. 368969]) with a hemolysis index (HI), measured by Abbott Diagnostics Architect c16000 platform, $\geq 1000$, corresponding to a free $\mathrm{Hb}$ concentration $\geq 10 \mathrm{~g} / \mathrm{L}$ [7]. Sixty patients had grossly hemolyzed samples, which accounted to $0.04 \%$ of the total $(n=166,752)$. The rate of gross hemolysis was significantly higher $(\mathrm{p}<0.0001$, $\chi^{2}$-test) in plasma samples ( $\mathrm{HI} \geq 1000$ in $0.13 \%$ of all plasma samples, $\mathrm{n}=34$ ) than that in serum samples ( $\mathrm{HI} \geq 1000$ in $0.02 \%$ of all serum samples, $n=26$ ). The higher rate of hemolysis in plasma samples in our setting can be, at least partly, explained by sample transportation with the pneumatic tube system previously shown to promote hemolysis in plasma specimens [8].

Table 1 shows the patient origin and stratification. Out of 60 patients with $\mathrm{HI} \geq 1000$, only one was outpatient. Six $(10.0 \%)$ were newborns in their first 3 days of life, two (3.3\%) were $<1$ year old and three $(5.5 \%)<10$ years old. No patients had diagnosis of in vivo hemolysis by their doctors at admission or during the hospitalization. Retrospectively, two of us (DS and SC) independently further assessed laboratory results and clinical data searching for any evidence of intravascular origin of gross hemolysis. In two pediatric emergency department patients, neither laboratory nor clinical data were sufficient for taking a definitive decision. Both (one infant $<2$ months old and a child aged 15 months) were quickly discharged because of a rapid improvement of their clinical condition. Anyway, after data matching and collegial discussion with assisting clinicians, it was not possible to diagnose an in vivo hemolysis condition in any of the recruited patients.

In conclusion, our data show that in vivo factors are involved in a very minority of cases of hemolysis, especially gross hemolysis. Therefore, our experimental findings support the EFLM WG-PRE recommendation advising the suppression of all test results in samples with $>10 \mathrm{~g} / \mathrm{L}$ of free $\mathrm{Hb}$ [1]. In laboratories where the assessment of $\mathrm{HI}$ is fully automated, the immediate transmission of hemolysis degree to the laboratory information system permits the test result suppression in grossly hemolized samples using computerized predefined rules [9]. We believe that this approach, in addition to being safe for the patient avoiding wrong interpretation of his tests, helps to speed up clinical path and decisions about the possible recollection of another blood sample.

There is an ongoing debate about how laboratories should deal with hemolyzed samples [10]. However, most of the articles focus on the question whether to suppress or report a single test result for analytes interfered by hemolysis. Beyond trying to answer to this question, the EFLM WG-PRE practical recommendations also deal with the issue of full sample rejection in case of gross hemolysis by proposing the suppression of all test results, including those analytes that are not directly interfered by the presence of hemolysis. The experience presented in this paper seems to fully support this approach. However, a dilemma remains unsolved: How to recognize an in vivo hemolysis in which the release of test results may become important? In our opinion, given the demonstrated infrequence of the joint presence of intravascular hemolysis and grossly hemolyzed samples, this may be solved only by a specific advice with the requesting clinician.

Table 1: Stratification of recruited patients with a blood sample with hemolysis index $(\mathrm{HI}) \geq 1000$ according to the probability of suffering of intravascular hemolysis.

\begin{tabular}{|c|c|c|c|c|}
\hline Sample collection site & No. (males) & $\begin{array}{l}\text { In vivo hemolysis ruled out } \\
\text { by laboratory data, } \mathrm{n}(\%)\end{array}$ & $\begin{array}{l}\text { In vivo hemolysis ruled } \\
\text { out by clinical data, } \mathrm{n}(\%)\end{array}$ & $\begin{array}{l}\text { In vivo hemolysis ruled out after data } \\
\text { matching and collegial discussion, } \mathrm{n}(\%)\end{array}$ \\
\hline \multicolumn{5}{|l|}{ Adults } \\
\hline Emergency department & $30(15)$ & $23(76.7)$ & $25(83.3)$ & $30(100)$ \\
\hline Intensive care unit & $1(0)$ & $1(100)$ & $1(100)$ & $1(100)$ \\
\hline Internal medicine ward & $16(7)$ & $15(93.8)$ & $10(62.5)$ & $16(100)$ \\
\hline Outpatient clinic & $1(0)$ & $1(100)$ & $1(100)$ & $1(100)$ \\
\hline Total & $48(22)$ & $40(83.3)$ & $37(77.1)$ & $48(100)$ \\
\hline \multicolumn{5}{|l|}{ Pediatrics } \\
\hline Emergency department & $4(2)$ & $0(0)$ & $2(50.0)$ & $4(100)$ \\
\hline Intensive care unit & $1(1)$ & $0(0)$ & $1(100)$ & $1(100)$ \\
\hline Internal medicine ward & $7(3)$ & $7(100)$ & $1(14.3)$ & $7(100)$ \\
\hline Total & $12(6)$ & $7(58.3)$ & $4(33.3)$ & $12(100)$ \\
\hline Grand total & $60(28)$ & $47(78.3)$ & $41(68.3)$ & $60(100)$ \\
\hline
\end{tabular}


Author contributions: All the authors have accepted responsibility for the entire content of this submitted manuscript and approved submission.

Research funding: None declared.

Employment or leadership: None declared.

Honorarium: None declared.

Competing interests: The funding organization(s) played no role in the study design; in the collection, analysis and interpretation of data; in the writing of the report; or in the decision to submit the report for publication.

\section{References}

1. Lippi G, Cadamuro J, von Meyer A, Simundic AM, on behalf of the European Federation of Clinical Chemistry and Laboratory Medicine (EFLM) Working Group for Preanalytical Phase (WG-PRE). Practical recommendations for managing hemolyzed samples in clinical chemistry testing. Clin Chem Lab Med 2018;56:718-27.

2. Lippi G, Blanckaert N, Bonini P, Green S, Kitchen S, Palicka V, et al. Haemolysis: an overview of the leading cause of unsuitable specimens in clinical laboratories. Clin Chem Lab Med 2008;46:764-72.
3. Barcellini W, Fattizzo B. Clinical applications of hemolytic markers in the differential diagnosis and management of hemolytic anemia. Dis Markers 2015;2015:635670.

4. Carraro P, Servidio G, Plebani M. Hemolyzed specimens: a reason for rejection or a clinical challenge? Clin Chem 2000;46:306-7.

5. Dolci A, Panteghini M. Harmonization of automated hemolysis index assessment and use: is it possible? Clin Chim Acta 2014;432:38-43.

6. Dolci A, Giavarina D, Pasqualetti S, Szőke D, Panteghini M. Total laboratory automation: do stat tests still matter? Clin Biochem 2017;50:605-11.

7. Aloisio E, Carnevale A, Pasqualetti S, Birindelli S, Dolci A, Panteghini M. Random uncertainty of photometric determination of hemolysis index on the Abbott Architect c16000 platform. Clin Biochem 2018;57:62-4.

8. Pasqualetti S, Szőke D, Panteghini M. Heparinate but not serum tubes are susceptible to hemolysis by pneumatic tube transportation. Clin Chem Lab Med 2016;54:785-9.

9. Clinical and Laboratory Standards Institute [CLSI]. Hemolysis, icterus, and lipemia/turbidity indices as indicators of interference in clinical laboratory analysis: approved guideline. CLSI document C56-A. Wayne, PA: Clinical and Laboratory Standards Institute, 2012.

10. Cadamuro J, Mrazek C, Haschke-Becher E, Sandberg S. To report or not to report: a proposal on how to deal with altered test results in hemolytic samples. Clin Chem Lab Med 2017;55:1109-11. 